\title{
Editorial of Special Issue of WISE 2019
}

\author{
Reynold Cheng ${ }^{1} \cdot$ Sibo Wang ${ }^{2} \cdot$ Wen Hua $^{3} \cdot$ Xin Huang $^{4}$
}

Published online: 18 August 2020

(c) The Author(s) 2020

We are pleased to present a special issue of Data Science and Engineering (DSE), which contains a collection of five papers from the WISE 2019 conference, and two regular submission papers.

The International Conference on Web Information Systems Engineering (WISE) has established itself as a community aiming at high-quality research and offering the ground for advancing efforts in topics related to Web information systems. WISE 2019 focuses on research, development, and applications in relation to Web information systems, including a wide range of topics, such as blockchain, deep learning, machine learning, recommender systems, data mining, Web-based applications, graph learning, knowledge graphs, graph mining, text mining, and crowdsourcing. WISE 2019 was held in Hong Kong, China, during January 19-21, 2020. WISE 2019 attracted a total of 211 research paper submissions. The conference program committee selected 50 full research papers (with an acceptance rate of 23.7\%), five demonstration papers, and three tutorial papers to be presented at the conference and published in the proceedings $[1,2]$. The conference program also included keynote presentations by Prof. Wei Zhao (American University of Sharjah, UAE) and Prof. Weiyi Meng (State University of New York at Binghamton, USA).

The five extended papers for this special issue were selected from among all the accepted papers by the special

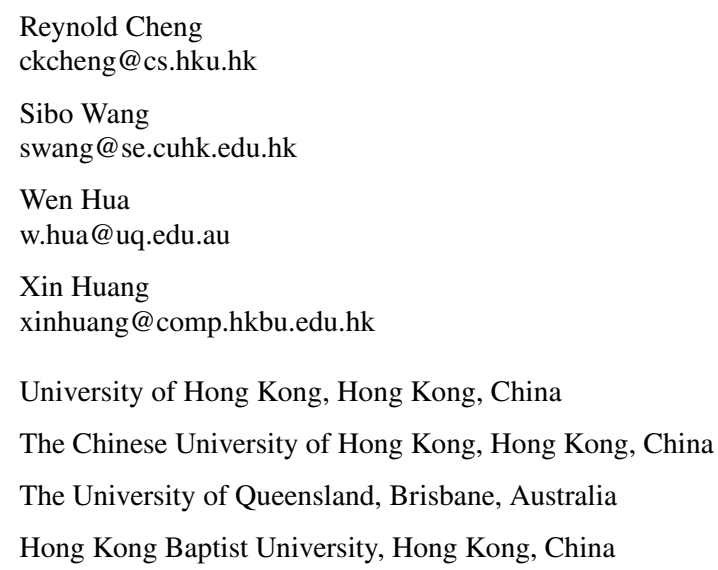

issue guest editors Reynold Cheng, Sibo Wang, Wen Hua, and Xin Huang, based on the relevance to the journal and the reviews of the conference version of the papers. The authors were asked to revise the conference paper for journal publication and in accordance with customary practice of adding $30 \%$ new materials. The revised papers again went through the review process in accordance with DSE guidelines and are finally presented to the readers in the present form.

The five extended papers in this special issue cover a variety of topics related to data science and engineering. The first paper "Feature-Based and Adaptive Rule Adaptation in Dynamic Environments" presented an autonomic and conceptual approach to adapt data annotation rules in unstructured and changing environments. The second paper "Real-Time Influence Maximization in a RTB Setting" proposed a real-time influence maximization approach to make influence maximization decisions within a real-time bidding environment. The third paper "Integrating Webbased Collaborative Live Editing and Wireframing into a Model-Driven Web Engineering Process" proposed a modeldriven Web engineering approach for microservice-based Web applications, which integrates the live code editing and wireframing capabilities. The fourth paper "An Interactive Network for End-to-end Review Helpfulness Modeling" proposed TRI, a deep interactive architecture to learn the text-rating interaction for end-to-end review helpfulness modeling. The last paper "Co-purchaser Recommendation for Online Group Buying" presented network embeddingbased methods to address the co-purchaser recommendation for online group buying.

We hope that the readers enjoy this special issue. We would like to acknowledge the work done by all the authors and their willingness to contribute their papers to this special issue. We thank all the reviewers for their expert comments and assistance in timely reviews. Finally, a note of thanks is to DSE Editors-in-Chief X. Sean Wang and Timos Sellis for their guidance and support in this process.

Reynold Cheng

Sibo Wang

Wen Hua 


\section{Xin Huang}

Open Access This article is licensed under a Creative Commons Attribution 4.0 International License, which permits use, sharing, adaptation, distribution and reproduction in any medium or format, as long as you give appropriate credit to the original author(s) and the source, provide a link to the Creative Commons licence, and indicate if changes were made. The images or other third party material in this article are included in the article's Creative Commons licence, unless indicated otherwise in a credit line to the material. If material is not included in the article's Creative Commons licence and your intended use is not permitted by statutory regulation or exceeds the permitted use, you will need to obtain permission directly from the copyright holder. To view a copy of this licence, visit http://creativecommons.org/licenses/by/4.0/.

\section{References}

1. Cheng R, Mamoulis N, Sun Y, Huang X (eds) (2019) Web information systems engineering-WISE 2019-20th international conference, Hong Kong, 26-30 Nov 2019, proceedings, volume 11881 of lecture notes in computer science. Springer

2. Yang J, Cai Y, Karlapalem K, Liu A, Huang X (eds) (2020) Web information systems engineering-WISE 2019 workshop, demo, and tutorial, Hong Kong and Macau, 19-22 Jan 2020, revised selected papers, volume 1155 of communications in computer and information science. Springer 